\title{
A Generic One-Factor Lévy Model for Pricing Synthetic CDOs
}

\author{
Hansjörg Albrecher ${ }^{1,2}$, Sophie A. Ladoucette ${ }^{3}$, and Wim Schoutens ${ }^{3}$ \\ 1 Johann Radon Institute \\ Austrian Academy of Sciences \\ Altenbergerstrasse 69 \\ A-4040 Linz, Austria \\ 2 Graz University of Technology \\ Department of Mathematics \\ Steyrergasse 30 \\ A-8010 Graz, Austria \\ albrecher@tugraz.at \\ 3 Katholieke Universiteit Leuven \\ Department of Mathematics \\ W. de Croylaan 54 \\ B-3001 Leuven, Belgium \\ sophie.ladoucette@wis.kuleuven.be, wim@schoutens.be
}

Summary. The one-factor Gaussian model is well-known not to fit simultaneously the prices of the different tranches of a collateralized debt obligation (CDO), leading to the implied correlation smile. Recently, other one-factor models based on different distributions have been proposed. Moosbrucker [12] used a one-factor Variance Gamma model, Kalemanova et al. [7] and Guégan and Houdain [6] worked with a NIG factor model and Baxter [3] introduced the BVG model. These models bring more flexibility into the dependence structure and allow tail dependence. We unify these approaches, describe a generic one-factor Lévy model and work out the large homogeneous portfolio (LHP) approximation. Then, we discuss several examples and calibrate a battery of models to market data.

\section{Introduction}

A collateralized debt obligation (CDO) can be defined as a transaction that transfers the credit risk on a reference portfolio of assets. A standard feature of a CDO structure is the tranching of credit risk. Credit tranching refers to creating multiple tranches of securities which have varying degrees of seniority and risk exposure. The risk of loss on the reference portfolio is then divided into tranches of increasing seniority in the following way. The equity tranche 
is the first to be affected by losses in the event of one or more defaults in the portfolio. If losses exceed the value of this tranche, they are absorbed by the mezzanine tranche(s). Losses that have not been absorbed by the other tranches are sustained by the senior tranche and finally by the supersenior tranche. Each tranche then protects the ones senior to it from the risk of loss on the underlying portfolio. When tranches are issued, they usually receive a rating by rating agencies. The CDO issuer typically determines the size of the senior tranche so that it is AAA-rated. Likewise, the CDO issuer generally designs the other tranches so that they achieve successively lower ratings. The CDO investors take on exposure to a particular tranche, effectively selling credit protection to the CDO issuer, and in turn collecting premiums (spreads).

We are interested in pricing tranches of synthetic CDOs. A synthetic CDO is a CDO backed by credit default swaps (CDSs) rather than bonds or loans, i.e. the reference portfolio is composed of CDSs. Recall that a CDS offers protection against default of an underlying entity over some time horizon. The term synthetic is used because CDSs permit synthetic exposure to credit risk. By contrast, a CDO backed by ordinary bonds or loans is called a cash CDO. Synthetic CDOs recently have become very popular.

It turns out that the pricing of synthetic CDO tranches only involves loss distributions over different time horizons (see Section 4). Then, we may think of using the large homogeneous portfolio (LHP) approximation to compute the premiums of these tranches. This convenient method is well-known in the credit portfolio field and permits to approximate the loss distribution which is computationally intensive.

Assuming a one-factor model approach for modeling correlated defaults of the different names in the reference portfolio together with the conditional independence of these defaults on a common market factor leads to a simplification of the calculation of the loss distribution (see Section 2). The one-factor Gaussian model is well-known not to fit simultaneously the prices of the different tranches of a CDO, leading to the implied correlation smile. Recently, other one-factor models based on different distributions have been proposed. Moosbrucker [12] used a one-factor Variance Gamma model, Kalemanova et al. [7] and Guégan and Houdain [6] worked with a NIG factor model and Baxter [3] introduced the BVG model. These models bring more flexibility into the dependence structure and allow tail dependence. We unify these approaches in Section 2, describe a generic one-factor Lévy model and work out the LHP approximation. With such a model, the distribution function of any name's asset value is analytically known, bringing significant improvement with respect to computation times. In Section 3, we discuss several examples, including the Gaussian, shifted Gamma, shifted Inverse Gaussian, Variance Gamma, Normal Inverse Gaussian and Meixner cases. Finally, we calibrate a battery of one-factor LHP Lévy models to market quotes of a tranched iTraxx and give the prices generated by these models. 
In 2004, the main traded CDS indices have been merged into a single family under the names DJ iTraxx (Europe and Asia) and DJ CDX (North America and emerging markets). These indices provide established portfolios upon which standardized tranches can be structured, allowing a more transparent and liquid market for CDO tranches.

Take the example of the DJ iTraxx Europe index. It consists of a portfolio composed of 125 actively traded names in terms of CDS volume, with an equal weighting given to each, and remains static over its lifetime of 6 months, except for entities defaulting which are then eliminated from the index. It is possible to invest in standardized tranches of the index via the tranched iTraxx which is nothing else but a synthetic CDO on a static portfolio. In other words, a tranched CDS index is a synthetic CDO based on a CDS index, where each tranche references a different segment of the loss distribution of the underlying CDS index. The main advantage of such a synthetic CDO relative to other CDOs is that it is standardized. In Table 1, we give the standard synthetic CDO structure on the DJ iTraxx Europe index.

Table 1. Standard synthetic CDO structure on the DJ iTraxx Europe index.

\begin{tabular}{|c|c|r|r|}
\hline Reference portfolio & Tranche name & $K_{1}$ & $K_{2}$ \\
\hline \multirow{3}{*}{125} & Equity & $0 \%$ & $3 \%$ \\
CDS & Junior mezzanine & $3 \%$ & $6 \%$ \\
names & Senior mezzanine & $6 \%$ & $9 \%$ \\
& Senior & $9 \%$ & $12 \%$ \\
& Super-senior & $12 \%$ & $22 \%$ \\
\hline
\end{tabular}

We end with some notation. By $f^{[-1]}$, we mean the inverse function of $f$. The indicator function of any set or event $A$ is denoted by $\mathbb{1}_{A}$. We use the abbreviation a.s. for almost surely and the symbol $\stackrel{a . s .}{\longrightarrow}$ stands for almost sure convergence. The gamma function is denoted by $\Gamma(x):=\int_{0}^{+\infty} t^{x-1} \mathrm{e}^{-t} \mathrm{~d} t$, $x>0$. The modified Bessel function of the third kind with real index $\zeta$ is denoted by $\mathrm{K}_{\zeta}(x):=\frac{1}{2} \int_{0}^{+\infty} t^{\zeta-1} \exp \left(-x\left(t+t^{-1}\right) / 2\right) \mathrm{d} t, x>0$. The notation $X \sim F$ means that the random variable $X$ follows the distribution $F$.

\section{Generic One-Factor Lévy Model}

\subsection{Lévy Process}

Suppose $\phi$ is the characteristic function of a distribution. If for every positive integer $n, \phi$ is also the $n$th power of a characteristic function, we say that the distribution is infinitely divisible. One can define for any infinitely divisible distribution a stochastic process, $X=\left\{X_{t}, t \geq 0\right\}$, called a Lévy process, which starts at zero, has stationary independent increments and such that 
the distribution of an increment over $[s, s+t], s, t \geq 0$, i.e. $X_{t+s}-X_{s}$, has $\phi^{t}$ as characteristic function.

The function $\psi:=\log \phi$ is called the characteristic exponent and it satisfies the following Lévy-Khintchine formula (see Bertoin [4]):

$$
\psi(z)=\mathrm{i} \gamma z-\frac{\varsigma^{2}}{2} z^{2}+\int_{-\infty}^{+\infty}\left(\exp (\mathrm{i} z x)-1-\mathrm{i} z x \mathbb{1}_{\{|x|<1\}}\right) \nu(\mathrm{d} x), \quad z \in \mathbb{R}
$$

where $\gamma \in \mathbb{R}, \varsigma^{2} \geq 0$ and $\nu$ is a measure on $\mathbb{R} \backslash\{0\}$ with $\int_{-\infty}^{+\infty} \min \left(1, x^{2}\right) \nu(\mathrm{d} x)<$ $\infty$. From the Lévy-Khintchine formula, one sees that, in general, a Lévy process consists of three independent parts: a linear deterministic part, a Brownian part, and a pure jump part. We say that our infinitely divisible distribution has a triplet of Lévy characteristics $\left[\gamma, \varsigma^{2}, \nu(\mathrm{d} x)\right]$. The measure $\nu(\mathrm{d} x)$ is called the Lévy measure of $X$ and it dictates how the jumps occur. Jumps of sizes in the set $A$ occur according to a Poisson process with parameter $\int_{A} \nu(\mathrm{d} x)$. If $\varsigma^{2}=0$ and $\int_{-1}^{+1}|x| \nu(\mathrm{d} x)<\infty$, it follows from standard Lévy process theory (e.g., Bertoin [4], Sato [16]) that the process is of finite variation. For more details about the applications of Lévy processes in finance, we refer to Schoutens [20].

\subsection{Generic One-Factor Lévy Model}

Next, we are going to model a portfolio of $n$ obligors; each obligor has the same weight in the portfolio. Later on, we will focus on a homogeneous portfolio, but let us start with the general situation where each obligor has some recovery value $R_{i}$ in case of default and some individual default probability term structure $p_{i}(t), t \geq 0$, which is the probability that obligor $i$ will default before time $t$.

Fix a time horizon $T$. For the modeling, let us start with a mother infinitely divisible distribution $L$. Let $X=\left\{X_{t}, t \in[0,1]\right\}$ be a Lévy process based on that infinitely divisible distribution, such that $X_{1}$ follows the law $L$. Note that we will only work with Lévy processes with time running over the unit interval. Denote the distribution function of $X_{t}$ by $H_{t}, t \in[0,1]$, and assume it is continuous. Assume further that the distribution is standardized in the sense that $\mathbb{E}\left[X_{1}\right]=0$ and $\operatorname{Var}\left[X_{1}\right]=1$. In terms of $\psi$, this means that $\psi^{\prime}(0)=0$ and $\psi^{\prime \prime}(0)=-1$. Then, it is not that hard to prove that $\operatorname{Var}\left[X_{t}\right]=t$.

Let $X=\left\{X_{t}, t \in[0,1]\right\}$ and $X^{(i)}=\left\{X_{t}^{(i)}, t \in[0,1]\right\}, i=1, \ldots, n$ be independent and identically distributed Lévy processes (so all processes are independent of each other and are based on the same mother infinitely divisible distribution $L$ ).

Next, we propose the generic one-factor Lévy model. Let $\rho \in(0,1)$. We assume that the asset value of obligor $i=1, \ldots, n$ at time $T$ is of the form:

$$
A_{i}(T)=X_{\rho}+X_{1-\rho}^{(i)} .
$$


Each $A_{i}(T)$ has by the stationary and independent increments property the same distribution as the mother distribution $L$ with distribution function $H_{1}$. Indeed, the sum of an increment of the process over a time interval of length $\rho$ and an independent increment over a time interval of length $1-\rho$ follows the distribution of an increment over an interval of unit length, i.e. is following the law $L$. As a consequence, $\mathbb{E}\left[A_{i}(T)\right]=0$ and $\operatorname{Var}\left[A_{i}(T)\right]=1$. Furthermore, the asset values of any two obligors $i$ and $j(i \neq j)$ are correlated with linear correlation coefficient $\rho$. Indeed, one readily computes:

$$
\begin{aligned}
\operatorname{Corr}\left[A_{i}(T), A_{j}(T)\right] & =\frac{\mathbb{E}\left[A_{i}(T) A_{j}(T)\right]-\mathbb{E}\left[A_{i}(T)\right] \mathbb{E}\left[A_{j}(T)\right]}{\sqrt{\operatorname{Var}\left[A_{i}(T)\right]} \sqrt{\operatorname{Var}\left[A_{j}(T)\right]}} \\
& =\mathbb{E}\left[A_{i}(T) A_{j}(T)\right]=\mathbb{E}\left[X_{\rho}^{2}\right]=\rho .
\end{aligned}
$$

So, starting from any mother standardized infinitely divisible law, we can set up a one-factor model with the required correlation.

We say that the $i$ th obligor defaults at time $T$ if its firm value $A_{i}(T)$ falls below some preset barrier $K_{i}(T)$, i.e. if $A_{i}(T) \leq K_{i}(T)$. In order to match default probabilities under this model with default probabilities $p_{i}(T)$ observed in the market, we have to set $K_{i}(T)=H_{1}^{[-1]}\left(p_{i}(T)\right)$. Indeed, it follows that $\mathbb{P}\left[A_{i}(T) \leq K_{i}(T)\right]=\mathbb{P}\left[A_{i}(T) \leq H_{1}^{[-1]}\left(p_{i}(T)\right)\right]=H_{1}\left(H_{1}^{[-1]}\left(p_{i}(T)\right)\right)=p_{i}(T)$.

Notice that conditional on the common factor $X_{\rho}$, the firm values and the defaults are independent.

From now on, we assume that the portfolio is homogeneous, i.e.:

- All obligors have the same default barrier $\left(K_{i}(T)=K(T), i=1, \ldots, n\right)$ and hence the same marginal default distribution $\left(p_{i}(T)=p(T)\right)$;

- All obligors have the same recovery rate $\left(R_{i}=R, i=1, \ldots, n\right)$;

- All obligors have the same notional amount: denoting the total portfolio notional by $N$, we then set $N_{i}=\frac{N}{n}$ for all $i=1, \ldots, n$.

Let us denote the number of defaults in the portfolio until time $T$ by $D_{T, n}$. The probability of having exactly $k$ defaults equals:

$$
\mathbb{P}\left[D_{T, n}=k\right]=\int_{-\infty}^{+\infty} \mathbb{P}\left[D_{T, n}=k \mid X_{\rho}=y\right] \mathrm{d} H_{\rho}(y), \quad k=0, \ldots, n .
$$

Conditional on $X_{\rho}=y$, the probability of having $k$ defaults is (because of independence):

$$
\mathbb{P}\left[D_{T, n}=k \mid X_{\rho}=y\right]=\left(\begin{array}{l}
n \\
k
\end{array}\right) p(y ; T)^{k}(1-p(y ; T))^{n-k}
$$

where $p(y ; T)$ denotes the probability that the firm's value $A_{i}(T)$ is below the barrier $K(T)$, given that the systematic factor $X_{\rho}$ takes the value $y$, i.e.: 


$$
\begin{aligned}
p(y ; T) & :=\mathbb{P}\left[A_{i}(T) \leq K(T) \mid X_{\rho}=y\right] \\
& =\mathbb{P}\left[X_{\rho}+X_{1-\rho}^{(i)} \leq K(T) \mid X_{\rho}=y\right] \\
& =\mathbb{P}\left[X_{1-\rho}^{(i)} \leq K(T)-y\right] \\
& =H_{1-\rho}(K(T)-y) .
\end{aligned}
$$

Substituting then yields:

$\mathbb{P}\left[D_{T, n}=k\right]=\left(\begin{array}{l}n \\ k\end{array}\right) \int_{-\infty}^{+\infty}\left(H_{1-\rho}(K(T)-y)\right)^{k}\left(1-H_{1-\rho}(K(T)-y)\right)^{n-k} \mathrm{~d} H_{\rho}(y)$.

The loss fraction on the portfolio notional at time $T$ given by:

$$
L_{T, n}^{\mathrm{HP}}:=\frac{1-R}{n} \sum_{i=1}^{n} \mathbb{1}_{\left\{A_{i}(T) \leq K(T)\right\}}
$$

is clearly one-to-one related with the number of defaults. We then obtain the following distribution function for the portfolio loss fraction:

$$
\mathbb{P}\left[L_{T, n}^{\mathrm{HP}} \leq \frac{k(1-R)}{n}\right]=\sum_{i=0}^{k} \mathbb{P}\left[D_{T, n}=i\right], \quad k=0, \ldots, n .
$$

In the next subsection, we will consider a method to approximate the latter distribution that turns out to be of prime interest in the CDO pricing (see Section 4).

Now, denote for small $x$ by $\lambda_{i j}(x):=\mathbb{P}\left[A_{j}(T) \leq x \mid A_{i}(T) \leq x\right], i \neq j$, a measure for the dependence in the tail. Using (conditional) independence arguments yields:

$$
\begin{aligned}
\lambda_{i j}(x) & =\frac{\mathbb{P}\left[X_{\rho}+X_{1-\rho}^{(j)} \leq x, X_{\rho}+X_{1-\rho}^{(i)} \leq x\right]}{\mathbb{P}\left[X_{\rho}+X_{1-\rho}^{(i)} \leq x\right]} \\
& =\frac{\mathbb{E}\left(\mathbb{P}\left[X_{\rho}+X_{1-\rho}^{(j)} \leq x, X_{\rho}+X_{1-\rho}^{(i)} \leq x \mid X_{\rho}\right]\right)}{H_{1}(x)} \\
& =\int_{-\infty}^{+\infty} \frac{\mathbb{P}\left[X_{1-\rho}^{(j)} \leq x-y, X_{1-\rho}^{(i)} \leq x-y\right]}{H_{1}(x)} \mathrm{d} H_{\rho}(y) \\
& =\int_{-\infty}^{+\infty} \frac{H_{1-\rho}^{2}(x-y)}{H_{1}(x)} \mathrm{d} H_{\rho}(y) .
\end{aligned}
$$

The limit $\lambda_{i j}:=\lim _{x \rightarrow-\infty} \lambda_{i j}(x)$ is then the well-known lower tail dependence coefficient of $A_{i}(T)$ and $A_{j}(T)$. Lower tail dependence is exactly capturing the probability of concordant down movements of the underlying asset values. The formula being stated in terms of the distribution $H_{t}$, the quantity $\lambda_{i j}(x)$ and its limit can be evaluated for any one-factor Lévy model. 


\subsection{The Large Homogeneous Portfolio Approximation}

We are interested in approximating the distribution function of the portfolio loss fraction $L_{T, n}^{\mathrm{HP}}$. This proves to be possible if the homogeneous portfolio gets very large (i.e. $n \rightarrow \infty$ ).

When conditioned on the systematic factor $X_{\rho}$, the default variables $\mathbb{1}_{\left\{A_{i}(T) \leq K(T)\right\}}, i=1, \ldots, n$, become independent. Hence, by the strong law of large numbers, we obtain:

$$
\mathbb{P}\left[\lim _{n \rightarrow \infty} L_{T, n}^{\mathrm{HP}}=(1-R) p\left(X_{\rho} ; T\right) \mid X_{\rho}\right]=1 \quad \text { a.s. }
$$

and taking expectations on both sides gives:

$$
L_{T, n}^{\mathrm{HP}} \stackrel{a . s .}{\longrightarrow}(1-R) p\left(X_{\rho} ; T\right) \quad \text { as } \quad n \rightarrow \infty .
$$

For large homogeneous portfolios, we then make the approximation:

$$
F_{T, n}^{\mathrm{HP}}(x):=\mathbb{P}\left[L_{T, n}^{\mathrm{HP}} \leq x\right]=F_{T}^{\mathrm{HP}}\left(\frac{x}{1-R}\right), \quad x \in[0,1-R]
$$

where $F_{T}^{\mathrm{HP}}$ denotes the distribution function of $p\left(X_{\rho} ; T\right)$, that turns out to be the loss fraction with a zero recovery rate (or equivalently the fraction of defaults) on the limiting portfolio at time $T$. We easily compute:

$$
\begin{aligned}
F_{T}^{\mathrm{HP}}(x) & =\mathbb{P}\left[H_{1-\rho}\left(K(T)-X_{\rho}\right) \leq x\right] \\
& =\mathbb{P}\left[X_{\rho} \geq K(T)-H_{1-\rho}^{[-1]}(x)\right] \\
& =1-H_{\rho}\left(H_{1}^{[-1]}(p(T))-H_{1-\rho}^{[-1]}(x)\right), \quad x \in[0,1]
\end{aligned}
$$

so that we obtain an explicit handy expression for the distribution $F_{T, n}^{\mathrm{HP}}$ of the portfolio fractional loss.

Note that if the portfolio contains a moderately large number of credits, the approximation (3) turns out to be remarkably good.

\section{Examples of One-Factor Lévy Models for Correlated Defaults}

\subsection{Based on the Normal Distribution - Brownian Motion}

The Vasicek [22] one-factor model assumes the following dynamics:

- $A_{i}(T)=\sqrt{\rho} Y+\sqrt{1-\rho} X_{i}, i=1, \ldots, n$;

- $Y, X_{i}, i=1, \ldots, n$ are i.i.d. standard normal random variables with common distribution function $\Phi$. 
This model can be casted in the above general Lévy framework. The mother infinitely divisible distribution is here the standard normal distribution and the associated Lévy process is the standard Brownian motion $W=\left\{W_{t}, t \in\right.$ $[0,1]\}$. Indeed, note that $W_{\rho}$ follows a $\operatorname{Normal}(0, \rho)$ distribution as does $\sqrt{\rho} Y$; similarly $W_{1-\rho}^{(i)}$ follows a $\operatorname{Normal}(0,1-\rho)$ distribution as does $\sqrt{1-\rho} X_{i}$. Adding these independent random variables leads to a standard normally distributed random variable.

Using the classical properties of normal random variables, the distribution function (4) of the fraction of defaulted securities in the limiting portfolio at time $T$ transforms into:

$$
\begin{aligned}
F_{T}^{\mathrm{HP}}(x) & =1-\Phi\left(\frac{\Phi^{[-1]}(p(T))-\sqrt{1-\rho} \Phi^{[-1]}(x)}{\sqrt{\rho}}\right) \\
& =\Phi\left(\frac{\sqrt{1-\rho} \Phi^{[-1]}(x)-\Phi^{[-1]}(p(T))}{\sqrt{\rho}}\right), \quad x \in[0,1] .
\end{aligned}
$$

\subsection{Based on the Shifted Gamma Process}

The density function of the Gamma distribution Gamma $(a, b)$ with parameters $a>0$ and $b>0$ is given by:

$$
f_{\text {Gamma }}(x ; a, b)=\frac{b^{a}}{\Gamma(a)} x^{a-1} \mathrm{e}^{-x b}, \quad x>0 .
$$

The distribution function of the $\operatorname{Gamma}(a, b)$ distribution is denoted by $\mathcal{H}_{G}(x ; a, b)$ and has the following characteristic function:

$$
\phi_{\text {Gamma }}(u ; a, b)=(1-\mathrm{i} u / b)^{-a}, \quad u \in \mathbb{R} .
$$

Clearly, this characteristic function is infinitely divisible. The Gamma process $X^{(G)}=\left\{X_{t}^{(G)}, t \geq 0\right\}$ with parameters $a, b>0$ is defined as the stochastic process which starts at zero and has stationary independent Gamma-distributed increments such that $X_{t}^{(G)}$ follows a $\operatorname{Gamma}(a t, b)$ distribution.

Table 2. Mean, variance, skewness and kurtosis of the $\operatorname{Gamma}(a, b)$ distribution.

\begin{tabular}{|c|c|}
\hline & Gamma $(a, b)$ \\
\hline mean & $a / b$ \\
variance & $a / b^{2}$ \\
skewness & $2 / \sqrt{a}$ \\
kurtosis & $3(1+2 / a)$ \\
\hline
\end{tabular}

The properties of the $\operatorname{Gamma}(a, b)$ distribution given in Table 2 can be easily derived from its characteristic function. Now, if $X$ is $\operatorname{Gamma}(a, b)$ distributed and $c>0$, then $c X$ is $\operatorname{Gamma}(a, b / c)$-distributed. Further, if 
$X \sim \operatorname{Gamma}\left(a_{1}, b\right)$ is independent of $Y \sim \operatorname{Gamma}\left(a_{2}, b\right)$ then $X+Y \sim$ $\operatorname{Gamma}\left(a_{1}+a_{2}, b\right)$.

Let us start with a Gamma process $G=\left\{G_{t}, t \in[0,1]\right\}$ with parameters $a>0$ and $b=\sqrt{a}$, so that $\mathbb{E}\left[G_{1}\right]=\sqrt{a}$ and $\operatorname{Var}\left[G_{1}\right]=1$. As driving Lévy process, we take the shifted Gamma process $X=\left\{X_{t}, t \in[0,1]\right\}$ defined as:

$$
X_{t}=\sqrt{a} t-G_{t}, \quad t \in[0,1] .
$$

The interpretation in terms of firm value is that there is a deterministic up trend $(\sqrt{a} t)$ with random downward shocks $\left(G_{t}\right)$.

The one-factor shifted Gamma-Lévy model is then in the form of (1):

$$
A_{i}(T)=X_{\rho}+X_{1-\rho}^{(i)}, \quad i=1, \ldots, n
$$

where $X_{\rho}, X_{1-\rho}^{(i)}, i=1, \ldots, n$ are independent shifted Gamma random variables defined as $X_{\rho}=\sqrt{a} \rho-G_{\rho}$ and $X_{1-\rho}^{(i)}=\sqrt{a}(1-\rho)-G_{1-\rho}$. By construction, each $A_{i}(T)$ follows the same distribution as $X_{1}$ and as such has zero mean and unit variance.

As derived in general, we have that the distribution of the limiting portfolio fractional loss with a zero recovery rate at time $T$ is as in (4). The distribution function $H_{t}(x ; a)$ of $X_{t}, t \in[0,1]$, can be easily obtained from the Gamma distribution function. Indeed:

$$
\begin{aligned}
H_{t}(x ; a) & =\mathbb{P}\left[\sqrt{a} t-G_{t} \leq x\right] \\
& =1-\mathbb{P}\left[G_{t}<\sqrt{a} t-x\right] \\
& =1-\mathcal{H}_{G}(\sqrt{a} t-x ; a t, \sqrt{a}), \quad x \in(-\infty, \sqrt{a} t) .
\end{aligned}
$$

For the inverse function, we have the following relation for each $t \in[0,1]$ :

$$
H_{t}^{[-1]}(y ; a)=\sqrt{a} t-\mathcal{H}_{G}^{[-1]}(1-y ; a t, \sqrt{a}), \quad y \in[0,1] .
$$

\subsection{Based on the Shifted IG Process}

The Inverse Gaussian $\operatorname{IG}(a, b)$ law with parameters $a>0$ and $b>0$ has characteristic function:

$$
\phi_{I G}(u ; a, b)=\exp \left(-a\left(\sqrt{-2 \mathrm{i} u+b^{2}}-b\right)\right), \quad u \in \mathbb{R} .
$$

The IG distribution is infinitely divisible and we define the IG process $X^{(I G)}=$ $\left\{X_{t}^{(I G)}, t \geq 0\right\}$ with parameters $a, b>0$ as the process which starts at zero and has stationary independent IG-distributed increments such that:

$$
\mathbb{E}\left[\exp \left(\mathrm{i} u X_{t}^{(I G)}\right)\right]=\phi_{I G}(u ; a t, b)=\exp \left(-a t\left(\sqrt{-2 \mathrm{i} u+b^{2}}-b\right)\right), \quad u \in \mathbb{R}
$$


meaning that $X_{t}^{(I G)}$ follows a IG(at,b) distribution.

The density function of the $\operatorname{IG}(a, b)$ law is explicitly known as:

$$
f_{I G}(x ; a, b)=\frac{a \mathrm{e}^{a b}}{\sqrt{2 \pi}} x^{-3 / 2} \exp \left(-\left(a^{2} x^{-1}+b^{2} x\right) / 2\right), \quad x>0
$$

and we denote its distribution function by $\mathcal{H}_{I G}(x ; a, b)$.

The characteristics of the IG distribution given in Table 3 can be easily obtained. Now, if $X$ is $\operatorname{IG}(a, b)$-distributed then $c X$ is $\operatorname{IG}(a \sqrt{c}, b / \sqrt{c})$-distributed for any positive $c$. Further, if $X \sim \operatorname{IG}\left(a_{1}, b\right)$ is independent of $Y \sim \operatorname{IG}\left(a_{2}, b\right)$ then $X+Y \sim \operatorname{IG}\left(a_{1}+a_{2}, b\right)$.

Table 3. Mean, variance, skewness and kurtosis of the $\operatorname{IG}(a, b)$ distribution.

\begin{tabular}{|c|c|}
\hline & $\mathrm{IG}(a, b)$ \\
\hline mean & $a / b$ \\
variance & $a / b^{3}$ \\
skewness & $3 / \sqrt{a b}$ \\
kurtosis & $3\left(1+5(a b)^{-1}\right)$ \\
\hline
\end{tabular}

Let us start with an IG process $I=\left\{I_{t}, t \in[0,1]\right\}$ with parameters $a>0$ and $b=a^{1 / 3}$, so that $\mathbb{E}\left[I_{1}\right]=a^{2 / 3}$ and $\operatorname{Var}\left[I_{1}\right]=1$. As driving Lévy process, we take the shifted IG process $X=\left\{X_{t}, t \in[0,1]\right\}$ defined as:

$$
X_{t}=a^{2 / 3} t-I_{t}, \quad t \in[0,1] .
$$

The interpretation in terms of firm value is that there is a deterministic up trend $\left(a^{2 / 3} t\right)$ with random downward shocks $\left(I_{t}\right)$.

The one-factor shifted IG-Lévy model is then:

$$
A_{i}(T)=X_{\rho}+X_{1-\rho}^{(i)}, \quad i=1, \ldots, n
$$

where $X_{\rho}, X_{1-\rho}^{(i)}, i=1, \ldots, n$ are independent shifted IG random variables defined as $X_{\rho}=a^{2 / 3} \rho-I_{\rho}$ and $X_{1-\rho}^{(i)}=a^{2 / 3}(1-\rho)-I_{1-\rho}$. Each $A_{i}(T)$ follows the same distribution as $X_{1}$ and as such has zero mean and unit variance.

The distribution of the fraction of defaulted securities in the limiting portfolio at time $T$ is therefore as in (4). The distribution function $H_{t}(x ; a)$ of $X_{t}$, $t \in[0,1]$, can be easily obtained from the IG distribution function as follows:

$$
\begin{aligned}
H_{t}(x ; a) & =\mathbb{P}\left[a^{2 / 3} t-I_{t} \leq x\right] \\
& =1-\mathbb{P}\left[I_{t}<a^{2 / 3} t-x\right] \\
& =1-\mathcal{H}_{I G}\left(a^{2 / 3} t-x ; a t, a^{1 / 3}\right), \quad x \in\left(-\infty, a^{2 / 3} t\right) .
\end{aligned}
$$

For the inverse function, we have the following relation for each $t \in[0,1]$ :

$$
H_{t}^{[-1]}(y ; a)=a^{2 / 3} t-\mathcal{H}_{I G}^{[-1]}\left(1-y ; a t, a^{1 / 3}\right), \quad y \in[0,1] .
$$




\subsection{Based on the VG Process}

The Variance Gamma (VG) distribution with parameters $\sigma>0, \nu>0, \theta \in \mathbb{R}$ and $\mu \in \mathbb{R}$, denoted by $\operatorname{VG}(\sigma, \nu, \theta, \mu)$, is infinitely divisible with characteristic function:

$$
\phi_{V G}(u ; \sigma, \nu, \theta, \mu)=\mathrm{e}^{\mathrm{i} u \mu}\left(1-\mathrm{i} u \theta \nu+u^{2} \sigma^{2} \nu / 2\right)^{-1 / \nu}, \quad u \in \mathbb{R} .
$$

We can then define the VG process $X^{(V G)}=\left\{X_{t}^{(V G)}, t \geq 0\right\}$ with parameters $\sigma, \nu>0$ and $\theta, \mu \in \mathbb{R}$ as the process which starts at zero and has stationary independent VG-distributed increments such that $X_{t}^{(V G)}$ follows a $\operatorname{VG}(\sigma \sqrt{t}, \nu / t, \theta t, \mu t)$ distribution. Note that a VG process may also be defined as a Brownian Motion with drift time-changed by a Gamma process (e.g., Schoutens [20]).

The density function of the $\operatorname{VG}(\sigma, \nu, \theta, \mu)$ distribution is given by:

$$
\begin{aligned}
f_{V G}(x ; \sigma, \nu, \theta, \mu)= & \frac{(G M)^{C}}{\sqrt{\pi} \Gamma(C)} \exp \left(\frac{(G-M)(x-\mu)}{2}\right) \\
& \times\left(\frac{|x-\mu|}{G+M}\right)^{C-1 / 2} K_{C-1 / 2}((G+M)|x-\mu| / 2), \quad x \in \mathbb{R}
\end{aligned}
$$

where $C, G, M$ are positive constants defined as:

$$
\begin{aligned}
C & :=1 / \nu \\
G & :=\left(\sqrt{\frac{\theta^{2} \nu^{2}}{4}+\frac{\sigma^{2} \nu}{2}}-\frac{\theta \nu}{2}\right)^{-1} \\
M & :=\left(\sqrt{\frac{\theta^{2} \nu^{2}}{4}+\frac{\sigma^{2} \nu}{2}}+\frac{\theta \nu}{2}\right)^{-1} .
\end{aligned}
$$

In Table 4, we give the values of the mean, variance, skewness and kurtosis of the $\operatorname{VG}(\sigma, \nu, \theta, \mu)$ distribution (the case $\theta=0$ is also included). This distribution is symmetric around $\mu$ if $\theta=0$ whereas negative values of $\theta$ result in negative skewness. Also, the parameter $\nu$ primarily controls the kurtosis.

Table 4. Mean, variance, skewness and kurtosis of the $\operatorname{VG}(\sigma, \nu, \theta, \mu)$ distribution.

\begin{tabular}{|c|c|c|}
\hline & $\mathrm{VG}(\sigma, \nu, \theta, \mu)$ & $\mathrm{VG}(\sigma, \nu, 0, \mu)$ \\
\hline mean & $\theta+\mu$ & $\mu$ \\
variance & $\sigma^{2}+\nu \theta^{2}$ & $\sigma^{2}$ \\
skewness & $\theta \nu\left(3 \sigma^{2}+2 \nu \theta^{2}\right) /\left(\sigma^{2}+\nu \theta^{2}\right)^{3 / 2}$ & 0 \\
kurtosis & $3\left(1+2 \nu-\nu \sigma^{4}\left(\sigma^{2}+\nu \theta^{2}\right)^{-2}\right)$ & $3(1+\nu)$ \\
\hline
\end{tabular}

The $\operatorname{VG}(\sigma, \nu, \theta, \mu)$ distribution satisfies the following scaling property: if $X \sim \operatorname{VG}(\sigma, \nu, \theta, \mu)$ then $c X \sim \operatorname{VG}(c \sigma, \nu, c \theta, c \mu)$ for all $c>0$. Also, we have the 
following convolution property: if $X \sim \operatorname{VG}(\sigma \sqrt{\rho}, \nu / \rho, \theta \rho, \mu \rho)$ is independent of $Y \sim \operatorname{VG}(\sigma \sqrt{1-\rho}, \nu /(1-\rho), \theta(1-\rho), \mu(1-\rho))$ then $X+Y \sim \operatorname{VG}(\sigma, \nu, \theta, \mu)$ under the constraint $\rho \in(0,1)$.

The class of VG distributions was introduced by Madan and Seneta [9]. A number of papers have developed the variance gamma model for asset returns and its implications for option pricing. In Madan and Seneta $[9,10]$ and Madan and Milne [8], the symmetric case $(\theta=0)$ is considered. In Madan et al. [11], the general case with skewness is treated. In equity and interest rate modelling, the VG process has already proven its capabilities, see e.g. Schoutens [20].

Moosbrucker assumes in [12] a one-factor VG model where the asset value of obligor $i=1, \ldots, n$ is of the form:

$$
A_{i}(T)=c Y+\sqrt{1-c^{2}} X_{i}
$$

where $Y, X_{i}, i=1, \ldots, n$ are independently VG-distributed random variables with $X_{i} \sim \operatorname{VG}\left(\sqrt{1-\nu \theta^{2}}, \nu /\left(1-c^{2}\right), \theta \sqrt{1-c^{2}},-\theta \sqrt{1-c^{2}}\right)$ for all $i$ and $Y \sim$ $\mathrm{VG}\left(\sqrt{1-\nu \theta^{2}}, \nu / c^{2}, \theta c,-\theta c\right)$. In this setting, the random variable $A_{i}(T)$ is $\mathrm{VG}\left(\sqrt{1-\nu \theta^{2}}, \nu, \theta,-\theta\right)$-distributed. All these random variables have indeed zero mean and unit variance but there is a constraint on the parameters, namely $\nu \theta^{2}<1$. Further, we point out that the fractional loss distribution obtained by Moosbrucker [12, Eq.(13) p.19] with the LHP method under the above one-factor model approach is not correct. It should be in his notation:

$$
F_{\text {portfolio loss }}(x)=1-F_{M}\left(\frac{C-\sqrt{1-c^{2}} F_{Z_{i}}^{-1}(x)}{c}\right)
$$

since the VG distribution function is not an even function if $(\theta, \mu) \neq(0,0)$.

For a variant of the VG model, extended with an additional normal factor, we refer to Baxter [3].

Many variations are possible. For example, one could start with a zero mean $\operatorname{VG}(\kappa \sigma, \nu, \kappa \theta,-\kappa \theta)$ distribution for $A_{i}(T)$, with $\kappa=1 / \sqrt{\sigma^{2}+\nu \theta^{2}}$ in order to force unit variance. The one-factor VG-Lévy model is then in the form of (1):

$$
A_{i}(T)=X_{\rho}+X_{1-\rho}^{(i)}, \quad i=1, \ldots, n
$$

where $X_{\rho}, X_{1-\rho}^{(i)}, i=1, \ldots, n$ are independent VG random variables with the following parameters: the common factor $X_{\rho}$ follows a distribution $\operatorname{VG}(\kappa \sqrt{\rho} \sigma$, $\nu / \rho, \kappa \rho \theta,-\kappa \rho \theta)$ and the idiosyncratic risks $X_{1-\rho}^{(i)}$ all follow a distribution $\operatorname{VG}(\kappa \sqrt{1-\rho} \sigma, \nu /(1-\rho), \kappa(1-\rho) \theta,-\kappa(1-\rho) \theta)$.

\subsection{Based on the NIG Process}

The Normal Inverse Gaussian (NIG) distribution with parameters $\alpha>0$, $\beta \in(-\alpha, \alpha), \delta>0$ and $\mu \in \mathbb{R}$, denoted by $\operatorname{NIG}(\alpha, \beta, \delta, \mu)$, has a characteristic 
function given by:

$\phi_{N I G}(u ; \alpha, \beta, \delta, \mu)=\exp \left(\mathrm{i} u \mu-\delta\left(\sqrt{\alpha^{2}-(\beta+\mathrm{i} u)^{2}}-\sqrt{\alpha^{2}-\beta^{2}}\right)\right), \quad u \in \mathbb{R}$.

We clearly see that this characteristic function is infinitely divisible. Hence, we can define the NIG process $X^{(N I G)}=\left\{X_{t}^{(N I G)}, t \geq 0\right\}$ with parameters $\alpha>0, \beta \in(-\alpha, \alpha), \delta>0$ and $\mu \in \mathbb{R}$ as the process which starts at zero and has stationary independent NIG-distributed increments such that $X_{t}^{(N I G)}$ is $\operatorname{NIG}(\alpha, \beta, \delta t, \mu t)$-distributed.

The NIG distribution was introduced by Barndorff-Nielsen [1]. See also Barndorff-Nielsen [2] and Rydberg [13, 14, 15]. Note that the density function of the $\operatorname{NIG}(\alpha, \beta, \delta, \mu)$ distribution is given for any $x \in \mathbb{R}$ by:

$$
f_{N I G}(x ; \alpha, \beta, \delta, \mu)=\frac{\alpha \delta}{\pi} \exp \left(\delta \sqrt{\alpha^{2}-\beta^{2}}+\beta(x-\mu)\right) \frac{K_{1}\left(\alpha \sqrt{\delta^{2}+(x-\mu)^{2}}\right)}{\sqrt{\delta^{2}+(x-\mu)^{2}}} .
$$

If a random variable $X$ is $\operatorname{NIG}(\alpha, \beta, \delta, \mu)$-distributed and $c>0$, then $c X$ is $\operatorname{NIG}(\alpha / c, \beta / c, c \delta, c \mu)$-distributed. If $\beta=0$, the distribution is symmetric around $\mu$. This can be seen from the characteristics of the NIG distribution given in Table 5. Further, if $X \sim \operatorname{NIG}\left(\alpha, \beta, \delta_{1}, \mu_{1}\right)$ is independent of $Y \sim$ $\operatorname{NIG}\left(\alpha, \beta, \delta_{2}, \mu_{2}\right)$ then $X+Y \sim \operatorname{NIG}\left(\alpha, \beta, \delta_{1}+\delta_{2}, \mu_{1}+\mu_{2}\right)$.

Table 5. Mean, variance, skweness and kurtosis of the $\operatorname{NIG}(\alpha, \beta, \delta, \mu)$ distribution.

\begin{tabular}{|c|c|c|}
\hline & $\operatorname{NIG}(\alpha, \beta, \delta, \mu)$ & $\operatorname{NIG}(\alpha, 0, \delta, \mu)$ \\
\hline mean & $\mu+\delta \beta / \sqrt{\alpha^{2}-\beta^{2}}$ & $\mu$ \\
variance & $\alpha^{2} \delta\left(\alpha^{2}-\beta^{2}\right)^{-3 / 2}$ & $\delta / \alpha$ \\
skewness & $3 \beta \alpha^{-1} \delta^{-1 / 2}\left(\alpha^{2}-\beta^{2}\right)^{-1 / 4}$ & 0 \\
kurtosis & $3\left(1+\frac{\alpha^{2}+4 \beta^{2}}{\delta \alpha^{2} \sqrt{\alpha^{2}-\beta^{2}}}\right)$ & $3\left(1+\delta^{-1} \alpha^{-1}\right)$ \\
\hline
\end{tabular}

Guégan and Houdain propose in [6] a factor model based on a NIGdistributed common factor but with standard normal idiosyncratic risks.

Kalemanova et al. [7] define a one-factor NIG model in the following way:

$$
A_{i}(T)=a Y+\sqrt{1-a^{2}} X_{i}, \quad i=1, \ldots, n
$$

where $Y, X_{i}, i=1, \ldots, n$ are independent NIG random variables with the following parameters: the common factor $Y$ follows a $\operatorname{NIG}\left(\alpha, \beta, \alpha,-\frac{\alpha \beta}{\sqrt{\alpha^{2}-\beta^{2}}}\right)$ distribution and the idiosyncratic risks $X_{i}$ all follow a $\operatorname{NIG}\left(\alpha \sqrt{1-a^{2}} / a\right.$, $\left.\beta \sqrt{1-a^{2}} / a, \alpha \sqrt{1-a^{2}} / a,-\frac{\alpha \beta}{\sqrt{\alpha^{2}-\beta^{2}}} \sqrt{1-a^{2}} / a\right)$ distribution. This leads to a $\operatorname{NIG}\left(\alpha / a, \beta / a, \alpha / a,-\frac{\alpha \beta}{a \sqrt{\alpha^{2}-\beta^{2}}}\right)$ distribution for $A_{i}(T)$. Note that this distribution does not have unit variance while the parameters $\delta$ and $\mu$ are fixed 
to obtain a zero mean distribution. Incidentally, we point out that the distribution of the fractional loss obtained by Kalemanova et al. [7, p.10] with the LHP approximation under the above one-factor model approach is not correct. It should be in their notation:

$$
F_{\infty}(x)=1-F_{\mathcal{N I \mathcal { G } ( 1 )}}\left(\frac{C-\sqrt{1-a^{2}} F_{\mathcal{N} \mathcal{G}\left(\frac{\sqrt{1-a^{2}}}{a}\right)}^{-1}(x)}{a}\right)
$$

since the NIG distribution function is not an even function if $(\beta, \mu) \neq(0,0)$.

Using our methodology, one can set up similar NIG models. E.g., let $X=\left\{X_{t}, t \in[0,1]\right\}$ be a NIG process, where $X_{1}$ follows a distribution $\operatorname{NIG}\left(\alpha, \beta,\left(\alpha^{2}-\beta^{2}\right)^{3 / 2} / \alpha^{2},-\left(\alpha^{2}-\beta^{2}\right) \beta / \alpha^{2}\right)$. Note that the parameters $\delta$ and $\mu$ are chosen such that $X_{1}$ has zero mean and unit variance. The one-factor NIG-Lévy model is then:

$$
A_{i}(T)=X_{\rho}+X_{1-\rho}^{(i)}, \quad i=1, \ldots, n
$$

where $X_{\rho}, X_{1-\rho}^{(i)}, i=1, \ldots, n$ are independently NIG-distributed random variables with $X_{1-\rho}^{(i)} \sim \operatorname{NIG}\left(\alpha, \beta,(1-\rho)\left(\alpha^{2}-\beta^{2}\right)^{3 / 2} / \alpha^{2},-(1-\rho)\left(\alpha^{2}-\beta^{2}\right) \beta / \alpha^{2}\right)$ for all $i$ and $X_{\rho} \sim \operatorname{NIG}\left(\alpha, \beta, \rho\left(\alpha^{2}-\beta^{2}\right)^{3 / 2} / \alpha^{2},-\rho\left(\alpha^{2}-\beta^{2}\right) \beta / \alpha^{2}\right)$. By construction, each $A_{i}(T)$ follows the same distribution as $X_{1}$.

\subsection{Based on the Meixner Process}

The density function of the Meixner distribution (Meixner $(\alpha, \beta, \delta, \mu))$ is given for any $x \in \mathbb{R}$ by:

$$
f_{\text {Meixner }}(x ; \alpha, \beta, \delta, \mu)=\frac{(2 \cos (\beta / 2))^{2 \delta}}{2 \alpha \pi \Gamma(2 \delta)} \exp \left(\frac{\beta(x-\mu)}{\alpha}\right)\left|\Gamma\left(\delta+\frac{\mathrm{i}(x-\mu)}{\alpha}\right)\right|^{2}
$$

where $\alpha>0, \beta \in(-\pi, \pi), \delta>0$ and $\mu \in \mathbb{R}$.

The characteristic function of the $\operatorname{Meixner}(\alpha, \beta, \delta, \mu)$ distribution is:

$$
\phi_{\text {Meixner }}(u ; \alpha, \beta, \delta, \mu)=\mathrm{e}^{\mathrm{i} u \mu}\left(\frac{\cos (\beta / 2)}{\cosh ((\alpha u-\mathrm{i} \beta) / 2)}\right)^{2 \delta}, \quad u \in \mathbb{R} .
$$

The Meixner $(\alpha, \beta, \delta, \mu)$ distribution being infinitely divisible, we can then associate with it a Lévy process which we call the Meixner process. More precisely, a Meixner process $X^{(\text {Meixner })}=\left\{X_{t}^{(\text {Meixner })}, t \geq 0\right\}$ with parameters $\alpha>0, \beta \in(-\pi, \pi), \delta>0$ and $\mu \in \mathbb{R}$ is a stochastic process which starts at zero and has stationary independent Meixner-distributed increments such that $X_{t}^{(\text {Meixner })}$ is Meixner $(\alpha, \beta, \delta t, \mu t)$-distributed. 
The Meixner process was introduced in Schoutens and Teugels [21]. See also Schoutens [17]. It was suggested to serve for fitting stock returns in Grigelionis [5]. This application in finance was worked out in Schoutens [18, 19].

In Table 6, we give some relevant quantities for the general case and the symmetric case around $\mu$, i.e. with $\beta=0$. Note that the kurtosis of any Meixner distribution is greater than that of the normal distribution. Now, if $X$ is Meixner $(\alpha, \beta, \delta, \mu)$-distributed and $c>0$, then $c X$ is $\operatorname{Meixner}(c \alpha, \beta, \delta, c \mu)$ distributed. Further, if $X \sim \operatorname{Meixner}\left(\alpha, \beta, \delta_{1}, \mu_{1}\right)$ is independent of $Y \sim$ $\operatorname{Meixner}\left(\alpha, \beta, \delta_{2}, \mu_{2}\right)$ then $X+Y \sim \operatorname{Meixner}\left(\alpha, \beta, \delta_{1}+\delta_{2}, \mu_{1}+\mu_{2}\right)$.

Table 6. Mean, variance, skweness and kurtosis of the Meixner $(\alpha, \beta, \delta, \mu)$ distribution.

\begin{tabular}{|c|c|c|}
\hline & Meixner $(\alpha, \beta, \delta, \mu)$ & Meixner $(\alpha, 0, \delta, \mu)$ \\
\hline mean & $\mu+\alpha \delta \tan (\beta / 2)$ & $\mu$ \\
variance & $\left(\cos ^{-2}(\beta / 2)\right) \alpha^{2} \delta / 2$ & $\alpha^{2} \delta / 2$ \\
skewness & $\sin (\beta / 2) \sqrt{2 / \delta}$ & 0 \\
kurtosis & $3+(2-\cos (\beta)) / \delta$ & $3+1 / \delta$ \\
\hline
\end{tabular}

Using our methodology, one can easily set up a Meixner model. E.g., let $X=\left\{X_{t}, t \in[0,1]\right\}$ be a Meixner process, where $X_{1}$ follows a distribution Meixner $\left(\alpha, \beta, 2 \cos ^{2}(\beta / 2) / \alpha^{2},-\sin (\beta) / \alpha\right)$. Note that again the parameters $\delta$ and $\mu$ are chosen such that $X_{1}$ has zero mean and unit variance. The one-factor Meixner-Lévy model is then:

$$
A_{i}(T)=X_{\rho}+X_{1-\rho}^{(i)}, \quad i=1, \ldots, n
$$

where $X_{\rho}, X_{1-\rho}^{(i)}, i=1, \ldots, n$ are independent Meixner random variables with the following parameters: the common factor $X_{\rho}$ follows a $\operatorname{Meixner}(\alpha, \beta$, $\left.2 \rho \cos ^{2}(\beta / 2) / \alpha^{2},-\rho \sin (\beta) / \alpha\right)$ distribution and the idiosyncratic risks $X_{1-\rho}^{(i)}$ all follow a Meixner $\left(\alpha, \beta, 2(1-\rho) \cos ^{2}(\beta / 2) / \alpha^{2},-(1-\rho) \sin (\beta) / \alpha\right)$ distribution. By construction, each $A_{i}(T)$ follows the same distribution as $X_{1}$.

\subsection{Other Candidate Models}

We hope the idea is clear and invite the reader to set up the CGMY, Generalized Hyperbolic (GH), Generalized $z$ (GZ) and other Lévy based models in a similar way. For definitions, we refer to Schoutens [20].

\section{Fair Pricing of a Synthetic CDO Tranche}

In this section, we explain the procedure for valuing the tranches of synthetic CDOs. Consider a synthetic CDO tranche on a given reference portfolio of $n$ 
names defined by an interval $\left[K_{1}, K_{2}\right]$ of loss fractions on the total portfolio notional $N$ that the tranche investor is responsible for. The endpoints $K_{1}$ and $K_{2}$ of the interval are called attachment and detachment points respectively. The tranche investor receives periodic spread payments from the CDO issuer (the premium leg) and makes payments to the CDO issuer when defaults affect the tranche (the protection leg). Note that for a synthetic CDO, any default corresponds to a credit event under a CDS in the reference portfolio. It turns out that the fair price of the tranche $\left[K_{1}, K_{2}\right]$ can be calculated using the same idea as for the pricing of a CDS, i.e. by setting the fair premium $s$ such that the expected present values of the premium leg and the protection leg are equal.

The loss fraction on the portfolio notional at time $t$ is given by:

$$
L_{t, n}:=\frac{1}{N} \sum_{i=1}^{n} \mathbb{1}_{\left\{\tau_{i} \leq t\right\}}\left(1-R_{i}\right) N_{i}
$$

where $\tau_{i}, R_{i}$ and $N_{i}$ denote the default time, the recovery rate and the notional amount of name $i$ respectively, $i=1, \ldots, n$. Under the factor model (1), we have $\left\{\tau_{i} \leq t\right\}=\left\{A_{i}(t) \leq K_{i}(t)\right\}$ with $K_{i}(t)=H_{1}^{[-1]}\left(p_{i}(t)\right), i=1, \ldots, n$.

The loss fraction on the CDO tranche $\left[K_{1}, K_{2}\right]$ at time $t$ is simply expressed by means of $L_{t, n}$ as:

$$
L_{t, n}\left(K_{1}, K_{2}\right):=\frac{\max \left\{\min \left(L_{t, n}, K_{2}\right)-K_{1}, 0\right\}}{K_{2}-K_{1}} .
$$

We assume that the payments (premium and protection legs) occur on periodic payment dates $t_{1}, \ldots, t_{m}$. Further, we assume that the CDO issuer receives compensation at the next scheduled payment date after a default has occurred. Note that payments are only made as long as the effective notional of the tranche at time $t_{i}$ is positive. Denote by $\tau_{(1)}, \ldots, \tau_{(n)}$ the order statistics, arranged in increasing order, of the random sample $\tau_{1}, \ldots, \tau_{n}$ of default times. Put $t_{0}:=0, \tau_{(0)}:=0$ and $L_{0, n}:=0$. In what follows, expectations are taken under a risk-neutral measure, i.e. risk-adjusted expectations.

The expected present value of the premium leg of the tranche is the present value of all spread payments the tranche investor expects to receive:

$$
\begin{aligned}
\mathrm{PL}(s)=s \mathbb{E}\left[\sum _ { j = 1 } ^ { m } \left\{\left(t_{j}-t_{j-1}\right) D\left(0, t_{j}\right)\left(1-L_{t_{j}, n}\left(K_{1}, K_{2}\right)\right)\right.\right. \\
+\sum_{i=1}^{n} \mathbb{1}_{\left\{t_{j-1}<\tau_{(i)}<t_{j}\right\}}\left(\tau_{(i)}-t_{j-1}\right) D\left(0, \tau_{(i)}\right) \\
\left.\left.\quad \times\left(L_{\tau_{(i)}, n}\left(K_{1}, K_{2}\right)-L_{\tau_{(i-1)}, n}\left(K_{1}, K_{2}\right)\right)\right\}\right]
\end{aligned}
$$

where $D\left(0, t_{j}\right)$ is the risk-free discount factor for payment date $t_{j}$ and $s$ is the spread per annum paid to the tranche investor. The term $1-L_{t_{j}, n}\left(K_{1}, K_{2}\right)$ 
is the fraction of the tranche notional outstanding on payment date $t_{j}$ and reflects the decline in notional as defaults affect the tranche. The second term in the sum over $j$ corresponds to the discounted sum of accrual payments the tranche investor receives when defaults occur between payment dates. They are paid at the next payment date and are based on the previous effective tranche notional.

The expected present value of the protection leg of the tranche is the discounted sum of the expected payments the tranche investor must make when defaults affect the tranche:

$\mathrm{LL}=\mathbb{E}\left[\sum_{j=1}^{m} D\left(0, t_{j}\right) \sum_{i=1}^{n} \mathbb{1}_{\left\{t_{j-1}<\tau_{(i)}<t_{j}\right\}}\left(L_{\tau_{(i)}, n}\left(K_{1}, K_{2}\right)-L_{\tau_{(i-1)}, n}\left(K_{1}, K_{2}\right)\right)\right]$.

The fair tranche premium $s_{\text {par }}$ is then the spread $s$ solving $\operatorname{PL}(s)=\mathrm{LL}$. As a consequence, the periodic payment received by the tranche investor from the CDO issuer in return for bearing the risk of losses is equal to $s_{\text {par }}$ times the effective outstanding notional of the tranche.

Now, assume a homogeneous portfolio. Recall that all obligors have the same default barrier $K_{i}(t)=K(t)$, the same recovery rate $R_{i}=R$ and the same notional amount $N_{i}=\frac{N}{n}$. Each of the $n$ obligors either takes no loss or a loss of $(1-R) \frac{N}{n}$ so that multiplying the number of defaults by $(1-R) \frac{N}{n}$ gives losses. Clearly, $L_{t, n}$ reduces to the loss fraction $L_{t, n}^{\mathrm{HP}}$ defined in (2). It follows that the expected loss fraction on the portfolio at time $t$ is:

$$
\mathbb{E}\left[L_{t, n}^{\mathrm{HP}}\right]=\frac{1-R}{n} \sum_{k=0}^{n} k \mathbb{P}\left[L_{t, n}^{\mathrm{HP}}=\frac{k(1-R)}{n}\right]
$$

and, denoting $L_{t, n}^{\mathrm{HP}}\left(K_{1}, K_{2}\right):=L_{t, n}\left(K_{1}, K_{2}\right)$, that the expected loss fraction on the CDO tranche $\left[K_{1}, K_{2}\right]$ at time $t$ is:

$$
\begin{aligned}
& \mathbb{E}\left[L_{t, n}^{\mathrm{HP}}\left(K_{1}, K_{2}\right)\right] \\
& \quad=\frac{1}{K_{2}-K_{1}} \sum_{k=0}^{n} \mathbb{P}\left[L_{t, n}^{\mathrm{HP}}=\frac{k(1-R)}{n}\right] \max \left\{\min \left(\frac{k(1-R)}{n}, K_{2}\right)-K_{1}, 0\right\}
\end{aligned}
$$

where $\mathbb{P}\left[L_{t, n}^{\mathrm{HP}}=\frac{k(1-R)}{n}\right]$ is the probability that exactly $k$ defaults occur by time $t, k=0, \ldots, n$.

As soon as the expected loss fraction on the tranche is calculated, the computation of the tranche premium becomes easy. Unfortunately, the derivation of the fractional loss distribution on the reference portfolio is not trivial. However, under the above homogeneity assumptions, we know from Subsection 2.3 that this distribution can be approximated using the LHP approximation method. As a consequence, this method provides an easy tool to compute both the fractional loss distribution and the expected loss fraction on the tranche over different time horizons. 
With the LHP approximation given in $(3)$, i.e. $F_{t, n}^{\mathrm{HP}}(x)=F_{t}^{\mathrm{HP}}(x /(1-R))$ for $x \in[0,1-R]$, we compute the expected loss fraction on the CDO tranche $\left[K_{1}, K_{2}\right]$ at time $t$ as:

$$
\begin{aligned}
& \mathbb{E}\left[L_{t, n}^{\mathrm{HP}}\left(K_{1}, K_{2}\right)\right] \\
& =\mathbb{E}\left[\frac{\max \left\{\min \left(L_{t, n}^{\mathrm{HP}}, K_{2}\right)-K_{1}, 0\right\}}{K_{2}-K_{1}}\right] \\
& =\mathbb{E}\left[\frac{\max \left(L_{t, n}^{\mathrm{HP}}-K_{1}, 0\right)-\max \left(L_{t, n}^{\mathrm{HP}}-K_{2}, 0\right)}{K_{2}-K_{1}}\right] \\
& =\frac{1}{K_{2}-K_{1}}\left(\int_{K_{1}}^{1-R}\left(x-K_{1}\right) \mathrm{d} F_{t, n}^{\mathrm{HP}}(x)-\int_{K_{2}}^{1-R}\left(x-K_{2}\right) \mathrm{d} F_{t, n}^{\mathrm{HP}}(x)\right) \\
& =\frac{1-R}{K_{2}-K_{1}}\left(\int_{\frac{K_{1}}{1-R}}^{1}\left(x-\frac{K_{1}}{1-R}\right) \mathrm{d} F_{t}^{\mathrm{HP}}(x)-\int_{\frac{K_{2}}{1-R}}^{1}\left(x-\frac{K_{2}}{1-R}\right) \mathrm{d} F_{t}^{\mathrm{HP}}(x)\right) .
\end{aligned}
$$

Similarly, the expected loss fraction on the last tranche $[K, 1]$ at time $t$ is given by:

$$
\begin{aligned}
\mathbb{E}\left[L_{t, n}^{\mathrm{HP}}(K, 1)\right] & =\mathbb{E}\left[\frac{\max \left(L_{t, n}^{\mathrm{HP}}-K, 0\right)}{1-K}\right] \\
& =\frac{1}{1-K} \int_{K}^{1-R}(x-K) \mathrm{d} F_{t, n}^{\mathrm{HP}}(x) \\
& =\frac{1-R}{1-K} \int_{\frac{K}{1-R}}^{1}\left(x-\frac{K}{1-R}\right) \mathrm{d} F_{t}^{\mathrm{HP}}(x)
\end{aligned}
$$

Finally, we report on a small calibration exercise of the Gaussian, shifted Gamma, shifted IG, VG and NIG cases. We calibrate the model to the iTraxx of the 4th of May 2006. In Table 7, one finds the market quotes together with the calibrated model quotes for the different tranches. Note that for the $0-3 \%$ tranche, the upfront is quoted with a 500 bp running.

Table 7. Pricing of iTraxx tranches of May 4, 2006 with LHP Lévy models.

\begin{tabular}{|c|r|r|r|r|r|r|}
\hline Model/Quotes & $0-3 \%$ & $3-6 \%$ & $6-9 \%$ & $9-12 \%$ & $12-22 \%$ & absolute error \\
\hline Market & $17 \%$ & $44.0 \mathrm{bp}$ & $12.8 \mathrm{bp}$ & $6.0 \mathrm{bp}$ & $2.0 \mathrm{bp}$ & \\
Gaussian & $17 \%$ & $105.7 \mathrm{bp}$ & $22.4 \mathrm{bp}$ & $5.7 \mathrm{bp}$ & $0.7 \mathrm{bp}$ & $73.7 \mathrm{bp}$ \\
Shifted Gamma & $17 \%$ & $44.0 \mathrm{bp}$ & $19.7 \mathrm{bp}$ & $11.9 \mathrm{bp}$ & $6.0 \mathrm{bp}$ & $16.8 \mathrm{bp}$ \\
Shifted IG & $17 \%$ & $44.0 \mathrm{bp}$ & $19.8 \mathrm{bp}$ & $12.2 \mathrm{bp}$ & $6.5 \mathrm{bp}$ & $17.7 \mathrm{bp}$ \\
VG & $17 \%$ & $43.9 \mathrm{bp}$ & $21.8 \mathrm{bp}$ & $14.1 \mathrm{bp}$ & $7.8 \mathrm{bp}$ & $23.0 \mathrm{bp}$ \\
NIG & $17 \%$ & $44.0 \mathrm{bp}$ & $24.1 \mathrm{bp}$ & $17.1 \mathrm{bp}$ & $11.7 \mathrm{bp}$ & $32.1 \mathrm{bp}$ \\
\hline
\end{tabular}




\section{Acknowledgments}

The authors thank Nomura and ING for providing the data. Special thanks go to Martin Baxter and Jürgen Tistaert. Hansjörg Albrecher is supported by the Austrian Science Fund Project P18392. Sophie A. Ladoucette is supported by the grant BDB-B/04/03 from the Catholic University of Leuven.

\section{References}

1. O.E. Barndorff-Nielsen. Normal inverse Gaussian distributions and the modelling of stock returns. Research Report No. 300, Department of Theoretical Statistics, Aarhus University, 1995.

2. O.E. Barndorff-Nielsen. Normal inverse Gaussian distributions and stochastic volatility modelling. Scandinavian Journal of Statistics, 24(1):1-13, 1997.

3. M. Baxter. Dynamic modelling of single-name credits and CDO tranches. Working Paper - Nomura Fixed Income Quant Group, 2006.

4. J. Bertoin. Lévy Processes. Cambridge Tracts in Mathematics 121. Cambridge University Press, Cambridge, 1996.

5. B. Grigelionis. Processes of Meixner type. Lithuanian Mathematical Journal, 39(1):33-41, 1999.

6. D. Guégan and J. Houdain. Collateralized Debt Obligations pricing and factor models: a new methodology using Normal Inverse Gaussian distributions. Research Report IDHE-MORA No. 07-2005, ENS Cachan, 2005.

7. A. Kalemanova, B. Schmid, and R. Werner. The Normal inverse Gaussian distribution for synthetic CDO pricing. Technical Report, 2005.

8. D.B. Madan and F. Milne. Option pricing with VG martingale components. Mathematical Finance, 1(4):39-55, 1991.

9. D.B. Madan and E. Seneta. Chebyshev polynomial approximations and characteristic function estimation. Journal of the Royal Statistical Society, Series B, 49(2):163-169, 1987.

10. D.B. Madan and E. Seneta. The Variance-Gamma (V.G.) model for share market returns. Journal of Business, 63(4):511-524, 1990.

11. D.B. Madan, P.P. Carr, and E.C. Chang. The Variance-Gamma process and option pricing. European Finance Review, 2:79-105, 1998.

12. T. Moosbrucker. Pricing CDOs with Correlated Variance Gamma Distributions. Research Report, Department of Banking, University of Cologne, 2006.

13. T. Rydberg. Generalized Hyperbolic Diffusions with Applications Towards Finance. Research Report No. 342, Department of Theoretical Statistics, Aarhus University, 1996.

14. T. Rydberg. The Normal Inverse Gaussian Lévy Process: Simulations and Approximation. Research Report No. 344, Department of Theoretical Statistics, Aarhus University, 1996.

15. T. Rydberg. A note on the existence of unique equivalent martingale measures in a Markovian setting. Finance and Stochastics, 1:251-257, 1997.

16. K. Sato. Lévy Processes and Infinitely Divisible Distributions. Cambridge Studies in Advanced Mathematics 68. Cambridge University Press, Cambridge, 2000.

17. W. Schoutens. Stochastic Processes and Orthogonal Polynomials. Lecture Notes in Statistics 146. Springer-Verlag, New York, 2000. 
18. W. Schoutens. The Meixner Process in Finance. EURANDOM Report 2001-002, EURANDOM, Eindhoven, 2001.

19. W. Schoutens. Meixner Processes: Theory and Applications in Finance. EURANDOM Report 2002-004, EURANDOM, Eindhoven, 2002.

20. W. Schoutens. Lévy Processes in Finance - Pricing Financial Derivatives. John Wiley \& Sons, Chichester, 2003.

21. W. Schoutens and J.L. Teugels. Lévy processes, polynomials and martingales. Communications in Statistics - Stochastic Models, 14(1-2):335-349, 1998.

22. O. Vasicek. Probability of Loss on Loan Portfolio. Technical Report, KMV Corporation, 1987. 